carcinoma) is also referred to as HCC (hepatocellular carcinoma) and hepatoma, and there are such sentences as "... ALC-associated OHC patients ..." (ALC = alcoholic liver cirrhosis), and "... HBV infection in PHC correlates with detection of HBsAg, $\mathrm{HBeAg}$ and HBV DNA ...". There are worse examples, which add to the confusion.

Finally, several criteria that should be satisfied in arguing the case for an aetiological role for hepatitis B virus in liver cancer were outlined in 1974: is the virus the driver or the passenger?; infection must precede development of the cancer; tumour cells should contain virus-specific molecules or antigens; the tumour cells should produce the agent; the virus should transform cells in culture or induce the tumour in animals; and immunisation should lower the incidence of the cancer (Zuckerman, A. J., Cell, 1, 1974). By and large the first four criteria have now been met. Maupas devoted the last few years of his life to initiating a comprehensive programme for the prevention of liver cancer by active immunisation against hepatitis B. Successful immunisation will provide, in the absence of an available model, the crucial evidence for an aetiological association. Much of the work of Professor Maupas is described in this volume, which is a fitting tribute to his memory. This short book is strongly recommended reading for hepatologists and virologists.

A. J. ZUCKERMAN

\title{
A colour atlas and textbook of the histopathology of mycotic diseases
}

By: F. W. Chandler, W. Kaplan and L. Ajello. 1980. Wolfe Medical Publications Ltd., London. Pp. 336. £35.

The excellence of this atlas lies in the more than 600 colour plates illustrating the various features of the pathogenic fungi in histological section. However, it is also a textbook and begins with chapters entitled "The taxonomy of the fungi and classification of fungal and actinomycotic diseases", "Histopathological diagnosis" and "Immunofluorescence diagnosiscurrent status". There then follow concise chapters on actinomycosis, adiaspiromycosis, aspergillosis, blastomycosis, candidiasis, chromoblastomycosis, coccidioidomycosis, cryptococcosis, dermatophilosis, histoplasmosis, lobomycosis, mycetomas, mycotic keratitis, nocardiosis, paracoccidioidomycosis, phaeohyphomycosis, protothecosis and infections caused by morphologically similar green algae, rare infections, rhinosporidiosis, sporotrichosis, superficial and cutaneous mycoses and zygomycosis. Each chapter is accompanied by carefully selected key references.

There are a few minor criticisms. Thus, in the chapter on superficial and cutaneous mycoses, the authors state that "Twenty-seven different species of dermatophyte are currently recognized", and table 1 includes Trichophyton verrucosum which is an important cause of cattle ringworm in Europe. On the other hand, in the chapter on taxonomy and classification 27 species of dermatophyte are listed, but here $T$. verrucosum is omitted and $T$. soudanense is included. The authors use the term candidiasis and state that "in the interest of clarity, brevity and uniformity we are coining the terms Histoplasmosis capsulati and Histoplasmosis farciminosi for infections caused by $H$. capsulatum var. capsulatum and $H$. farcininosum, respectively. The precedent for this nomenclature was set in 1964 by Cockshott and Lucas when they created the name histoplasmosis duboisii for infections by $H$. capsulatum var duboisii". However, the International Society for Human and Animal Mycology (Sabouraudia 1980, 18, 78-84) has recommended use of the terms candidosis for yeast infections by Candida spp. and classical histoplasmosis (American histoplasmosis) and African histoplasmosis for the diseases due to Histoplasma capsulatum and Histoplasma duboisi, respectively. In view of these recommendations, a disease name that is part vernacular and part specific epithet, from the latin name of the organism, is unlikely to find widespread acceptance.

I would like to have seen better illustrations of some of the cellular responses, but the deficiencies may be the result of the colour-reproduction processes used. Some of the plates show histological sections of fungi in animal tissues and the atlas will, therefore, also be of value to the veterinary pathologist. 
This book is a Wolfe Medical Publications companion to the Colour Atlas of Pathogenic Fungi by Frey, Oldfield and Bridger, (reviewed in this Journal, 13, p. 616) and is highly commended to histopathologists and microbiologists who need to identify fungi in tissue sections.

R. R. DAVIES 